\title{
Collimation and characterization of ELI-NP gamma beam
}

\author{
G. Cappello ${ }^{1, *}$, O. Adriani ${ }^{5,6}$, S. Albergo ${ }^{1,2}$, M. Andreotti ${ }^{3}$, D. Berto ${ }^{4}$, R. Borgheresi ${ }^{5,6}$, P. Cardarelli ${ }^{3}$, \\ R. Ciaranfi ${ }^{5}$, E. Consoli ${ }^{3}$, G. Di Domenico ${ }^{3}$, F. Evangelisti ${ }^{3}$, M. Gambaccini ${ }^{3,4}$, G. Graziani ${ }^{5}$, M. Lenzi ${ }^{5}$, \\ M. Marziani ${ }^{3}$, L. Palumbo ${ }^{8,9}$, G. Passaleva ${ }^{5}$, G. Paternò ${ }^{3}$, M. G. Pellegriti ${ }^{1}$, A. Serban ${ }^{5,11}$, O. Starodubtsev ${ }^{5}$, \\ M. Statera ${ }^{3}$, A. Tricomi ${ }^{1,2}$, A. Variola ${ }^{7}$ and M. Veltri ${ }^{5,10}$ \\ ${ }^{1}$ Istituto Nazionale di Fisica Nucleare, INFN - Sezione di Catania, via S.Sofia, 64, Catania, Italy \\ "corresponding author: gigi.cappello@ct.infn.it \\ ${ }^{2}$ Dipartimento di Fisica e Astronomia, Università di Catania, Catania, Italy \\ ${ }^{3}$ Istituto Nazionale di Fisica Nucleare, INFN - Sezione di Ferrara, via Saragat, 1, Ferrara, Italy \\ ${ }^{4}$ Dipartimento di Fisica e Scienze della Terra, Università di Ferrara, Ferrara, Italy \\ ${ }^{5}$ Istituto Nazionale di Fisica Nucleare, INFN - Sezione di Firenze, via Sansone, 1, Sesto F., Italy \\ ${ }^{6}$ Dipartimento di Fisica e Astronomia, Università di Firenze, Firenze, Italy \\ ${ }^{7}$ Istituto Nazionale di Fisica Nucleare, INFN - Laboratori Nazionali di Frascati, Frascati, Italy \\ ${ }^{8}$ Istituto Nazionale di Fisica Nucleare, INFN - Sezione di Roma, Roma, Italy \\ ${ }^{9}$ Dipartimento di Scienze di Base e Applicate all'Ingegneria, Università di Roma La Sapienza, Roma, Italy \\ ${ }^{10}$ Dipartimento di Scienze Pure e Applicate, Università di Urbino, Urbino, Italy \\ ${ }^{11}$ on leave from National Institute for Nuclear Physics and Engineering, Magurele, Romania
}

Published 10 July 2018

\begin{abstract}
The ELI-NP facility, currently being built in Bucharest, Romania, will deliver an intense and almost monochromatic gamma beam with tunable energy between 0.2 and $20 \mathrm{MeV}$. The challenging energy bandwidth of $\leq 0.5 \%$ will be adjusted through the collimation system, while the main beam parameters will be measured through a devoted gamma-beam characterization system. ${ }^{1}$ The gamma-beam characterization system, designed by the EuroGammaS collaboration, consists of four elements: a Compton spectrometer that measures the gamma energy spectrum; a sampling calorimeter for a fast combined measurement of the beam average energy and its intensity, which will be used also as a monitor during machine commissioning and development; a nuclear resonant scattering system for absolute energy inter-calibration of the other detectors; and a gamma beam profile imager to be used for alignment and diagnostics purposes. The collimation and characterization system will be presented in this article. These systems have already been built and tested, while the delivery at ELI-NP facility and the final commissioning is scheduled by Fall 2018 .
\end{abstract}

Keywords: Gamma beam; collimation; characterization.

\section{Introduction}

ELI (Extreme Light Infrastructure) is a project involving over 40 institutions from 13 European countries. High-power lasers and intense beam lines will be provided at three sites in Szeged, Hungary; Prague, Czech Republic; and Bucharest, Romania. The last one, ELI-NP, ${ }^{2}$ will be devoted to Nuclear Physics research. The research activities foreseen for the ELI-NP experiments ${ }^{3}$ (such as nuclear resonance fluorescence studies, measurements of the cross sections of photonuclear processes, studies on photo-fission,

This is an Open Access article published by World Scientific Publishing Company. It is distributed under the terms of the Creative Commons Attribution 4.0 (CC-BY) License. Further distribution of this work is permitted, provided the original work is properly cited. 
and expoloration of nuclear collective excitation models) require a brilliant, nearly monochromatic, and highly polarized gamma beam. The design, manufacturing, delivery, installation, testing, commissioning, and maintenance of the Gamma Beam System (GBS) is provided by the EuroGammaS consortium, an association of Italian and French institutions led by the Italian Institute of Nuclear Physics (INFN). The GBS ${ }^{4}$ will produce an intense $\gamma$-beam from Compton inverse scattering of a $515 \mathrm{~nm}$ pulsed laser light from an electron beam with tunable energy between 80 and $720 \mathrm{MeV}$. This will turn into a $\gamma$ beam energy ranging between $0.2 \mathrm{MeV}$ and $19.5 \mathrm{MeV}$. The laser-beam interaction leads to a peculiar time structure for the final $\gamma$-beam that will be made of macro-pulses, with a $100 \mathrm{~Hz}$ repetition rate and duration of $496 \mathrm{~ns}$, each containing a package of 32 micropulses separated by 16 ns. Every micro-pulse will contain $\sim 10^{5}$ photons. Most of the beam parameters represent a clear step forward with respect to the state-of-the-art technology. ${ }^{1}$ Among the beam parameters is a peak brilliance of $10^{20}-10^{23} \mathrm{\gamma} / \mathrm{s}^{\mathrm{mm}} \mathrm{m}^{2} \cdot \mathrm{mrad}^{2}$, a linear polarization $\geq 99 \%$, and a bandwidth of $\leq 0.5 \%$ that can be achieved using an efficient, well-designed collimation system. A precise energy calibration of the $\gamma$-beam and continuous monitoring of its key parameters (peak energy, space and time profile, intensity, etc.) during its operation are also necessary. Given the unprecedented characteristics of the beam, these tasks are themselves an experimental challenge.

Within the EuroGammaS collaboration, the design, building, testing, commissioning and maintenance of the characterization and collimation systems are conducted by the work package 9 (WP09), involving researchers from universities and INFN sections of Catania, Ferrara and Florence (Italy). The following sections include a brief description of the design of the collimator and the beam-characterization system, as well as details of the simulation studies and the preliminary tests performed on any subsystem.

\section{Overview of the System}

Two complete systems will be delivered for the two $\gamma$-beam lines of ELI-NP: the lowenergy (LE) line and the high-energy (HE) line. The two systems will be almost identical, except for minor design and experimental solutions optimized for the different energy ranges. An overall view of the LE line system is shown in Fig. 1.

A $100 \mathrm{~cm}$ thick concrete shielding wall is placed between the collimator (first element in the left part of the Fig. 1) and the characterization system (GBC), which is made up of the following four subsystems (from left to right, along the beam direction):

- A Compton spectrometer, which will provide precise peak-energy measurements while ensuring minimal interference with the beam operation;

- A nuclear resonant scattering system, which will provide an absolute energy calibration and a time reference to the other subsystems;

- A beam profile imager, which will determine the spatial profile of the beam downstream from the collimator;

- A sampling calorimeter, which will provide fast measurements of the total beam energy and the photon average energy, thus enabling measurement of the beam intensity. 


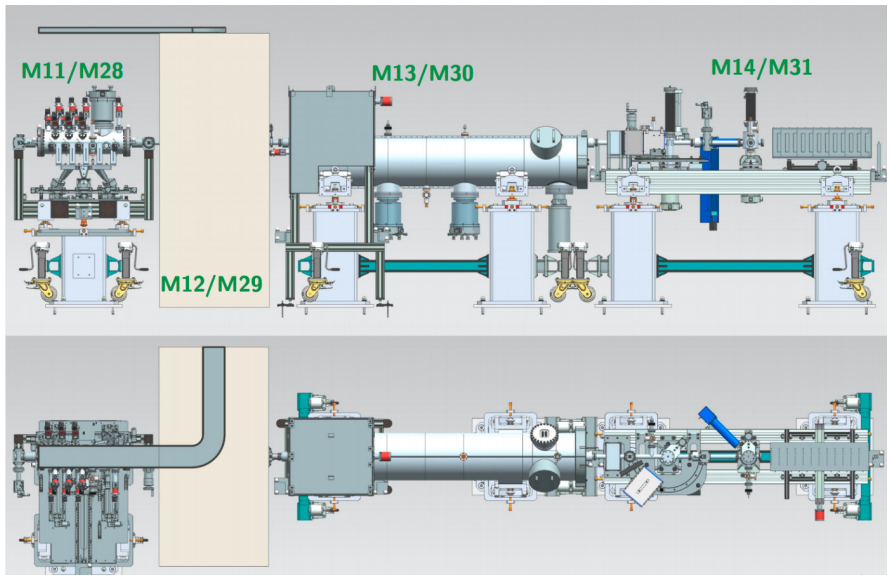

Fig. 1. Side and top views of the LE collimation and beam-characterization system of the ELI-NP-GBS. From left to right: the collimation (M11/M28 area) followed by a concrete wall (M12/M29 area); the Compton spectrometer (CSPEC, M13/M30); the NRS system, the GPI and the sampling calorimeter (M14/M31 area).

A detailed model of the whole collimation and GBC has been developed in Geant4 (a software toolkit for the simulation of the passage of particles through matter). Simulation studies drove the choice of the design and experimental solutions for all the subsystems. Realistic $\gamma$-beams have been used as primaries for both LE and HE beam conditions. ${ }^{5}$ These beams were obtained by running a transport model of $\mathrm{e}^{-}$bunches to the Compton interaction point and then simulating collisions with the laser pulses using the CAIN code. ${ }^{6}$

\section{The Collimation System (GCOLL)}

The collimation system ${ }^{7}$ (Fig. 1 on the left and Fig. 2 in detail) consists of a stack of 14 slits independently adjustable in aperture (from 0 to $25 \mathrm{~mm}$ ), each consisting of a pair of $4 \mathrm{~cm} \times 4 \mathrm{~cm} x 2 \mathrm{~cm}$ tungsten blocks manufactured with a roughness of $\leq 5 \mu \mathrm{m}$. The first 12 slits are arranged in three groups of four slits each with a relative rotation of $45^{\circ} \pm 0.15^{\circ}$ around the beam axis. The choice of the material was a compromise between high density and weight and was driven by the necessity to reduce the transmission of external photons, simultaneously ensuring precise movements of the slits. Studies of the production of secondary radiation were also conducted using detailed Geant 4 simulations to optimize the design and screening of the systems beyond the collimator. The whole system will be placed inside a vacuum chamber (pressure $\sim 10^{-7} \mathrm{mbar}$ ).

The critical physics requirement for GCOLL is to ensure a bandwidth of $\leq 0.5 \%$ throughout the energy range. In practice, this turns into a required collimation at 70-700 $\mu \mathrm{rad}$, corresponding in the adopted design to slit apertures continuously and precisely adjustable (with an accuracy of $\sim 40 \mu \mathrm{m}$ ) between $1 \mathrm{~mm}$ and $14 \mathrm{~mm}$. The effects of collimation on the bandwidth beams of different energies have been simulated in Geant4; they are shown in Fig. 3 for a beam with maximum energy $10 \mathrm{MeV}$ at interaction point, 
where the achievement of the $0.5 \%$ bandwidth goal can be seen. Figure 4 shows how a slight variation in aperture affects the intensity and bandwidth of a $3 \mathrm{MeV}$ beam. This effect is more pronounced at higher energy, thus reinforcing the need for micrometric building and adjustment precision. The determination of the mechanical tolerances were performed while studying the effects of misalignments of the slits and the chamber with respect to the beam axis.



Fig. 2. Design of the collimation system.
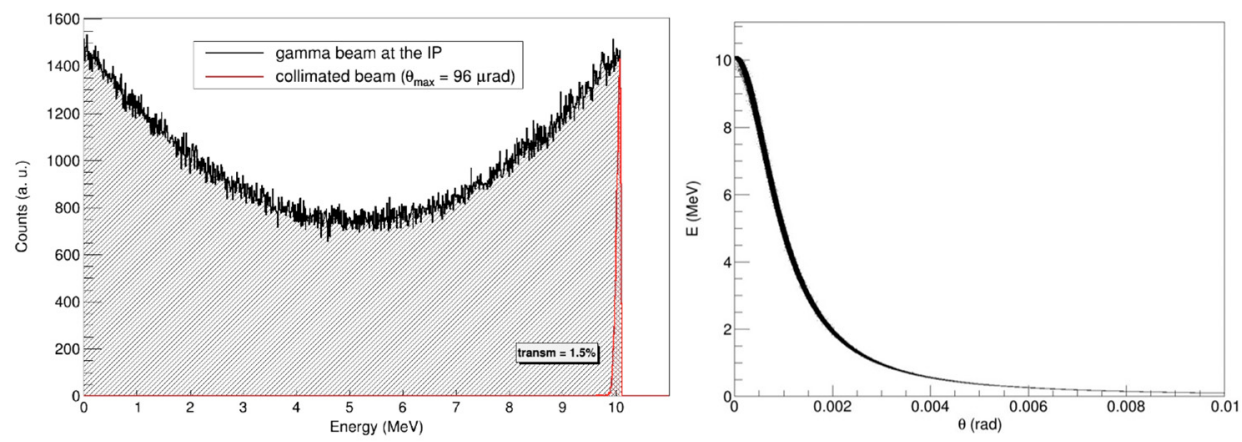

Fig. 3. (left) The $\gamma$-energy spectrum at Compton interaction point (black) and the collimated spectrum (red). (right) Beam energy distribution as function of the collimation angle.

\section{The Gamma Beam Characterization System}

The key parameters of the ELI-NP $\gamma$-beam (such as peak energy, bandwidth, space-andtime profile, and beam intensity) are measured and monitored using a Gamma Beam Characterization system (GBC). The GBC is based on the interplay of four different subsystems and on complementary approaches to and redundancy of the measurement of the same parameters. Details of every subsystem will be presented in the following sections. 


\section{Collimated Beam Spectrum @ $3 \mathrm{MeV}$}

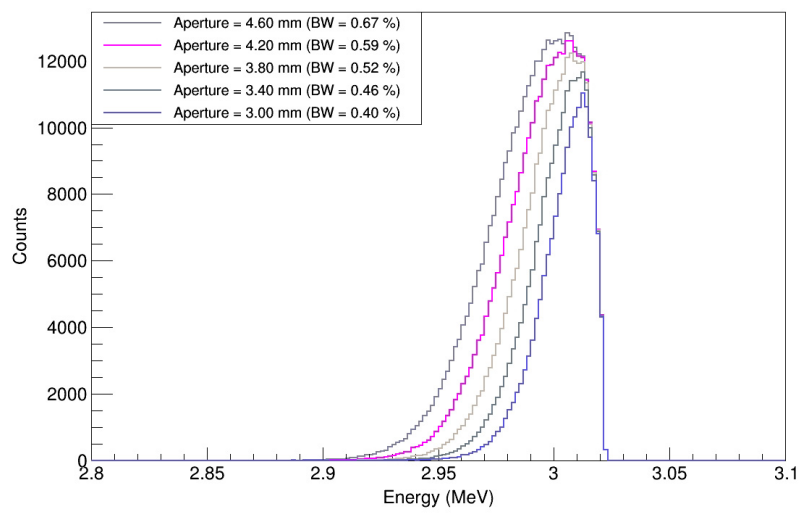

Fig. 4. Effects of variations of slits aperture on beam bandwidth and intensity. The bandwidth moves from $0.4 \%$ to $0.67 \%$ when the slits aperture varies from $3.0 \mathrm{~mm}$ to $4.6 \mathrm{~mm}$.

\subsection{Compton spectrometer (CSPEC)}

The Compton spectrometer (CSPEC, see Fig. 5) will reconstruct the $\gamma$-energy spectrum by measuring the energy and position of electrons recoiling at small angles from Compton interactions of the beam on a thin $(1-100 \mu \mathrm{m})$ mylar target. At small polar angles, the electrons carry most of the incident $\gamma$ energy (Eq. (1)), and the uncertainty in energy related to the lack of knowledge in the exact angle (due to multiple scattering and the transverse beam size) is limited.

$$
E_{\text {beam }}=\frac{m_{e} T_{e}}{\cos \phi \sqrt{T_{e}\left(T_{e}+2 m_{e}\right)}-T_{e}} .
$$

The background from pair production can be reduced by detecting the scattered photon in coincidence, whose energy and position are known from theory once the electron is measured. For this purpose, a $\gamma$-detector made of a matrix of barium fluoride crystals will be used. The electron energy will be measured with a custom-designed hyper-pure germanium crystal (HPGe) chosen for its extraordinary energy resolution ( $\sim 0.1 \%$ at $\mathrm{MeV}$ energies) and high detection efficiency. The HPGe is a planar detector (20 mm thick with $80 \mathrm{~mm}$ diameter with $<1 \mu \mathrm{m}$ dead layer) placed at $\sim 2 \mathrm{~m}$ from the target. Given that the time needed to perform a measurement is on the same order of magnitude as that of a macro-pulse length, the expected signal rate must be reduced to $<100 \mathrm{~Hz}$. This result can be obtained using micrometric Mylar targets, leading to a rejection factor of $\mathrm{O}\left(10^{6}\right)$. This choice also has the advantage of reducing the effects of multiple scattering and (most importantly) interference with the $\gamma$-beam. The ability to use the system for beam characterization during the commissioning and for monitoring during operation of the ELI-NP beam is one of the key features of the CSPEC.

The impact positions of electrons (and hence their angles) is finally measured with a $300-\mu \mathrm{m}$-thick double-sided silicon strip detector placed in front of the HPGe. The 
detector allows a two-dimensional reconstruction of the electron impact point with a resolution of $50 \mu \mathrm{m}$. A schematic view of the CSPEC and its working principle is shown in Fig. 5.
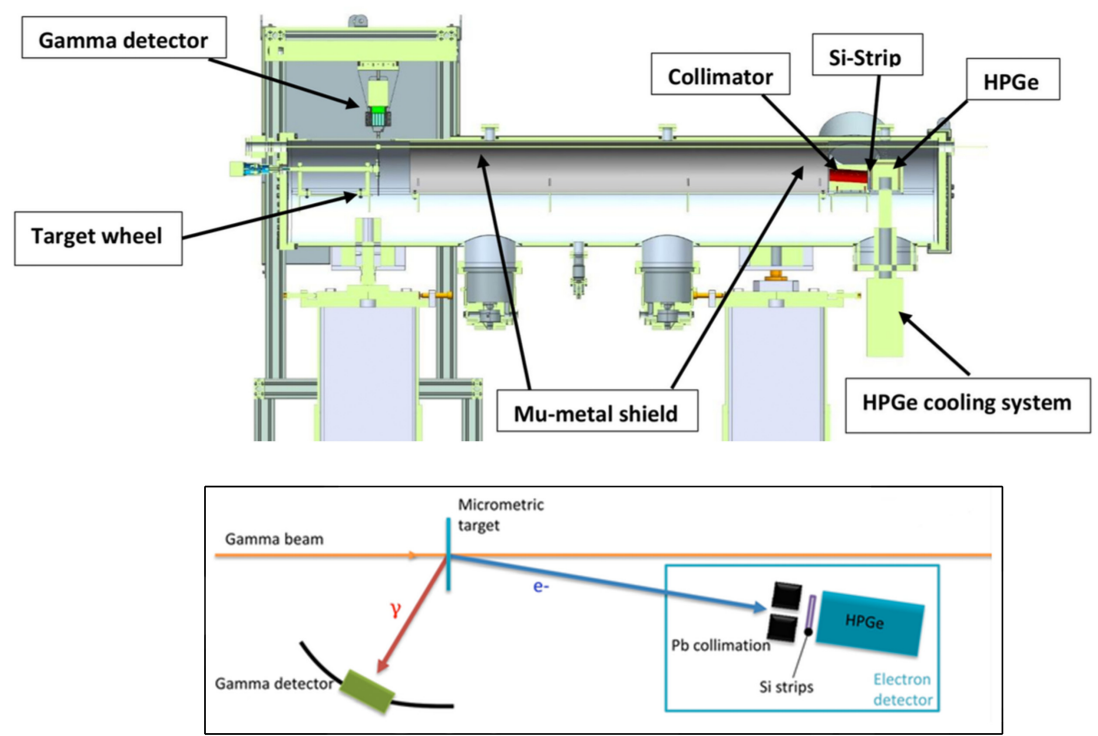

Fig. 5. The CSPEC and its working principle.

The expected performances ${ }^{8}$ were evaluated by simulating the detector and surrounding material for 100 -second data takings $\left(3.2 \times 10^{10} \gamma\right)$ at different energies, demonstrating that the peak energy can be reconstructed within $0.1 \%$ uncertainty and the energy profile of the beam can be reconstructed with an uncertainty in the bandwidth of $10-40 \%$. Resolution and linearity tests were recently conducted in Florence with different radioactive sources. A $\mathrm{FWHM} / \mathrm{E}=0.167 \%$ has been measured for the ${ }^{60} \mathrm{Co}$ line at $1173 \mathrm{keV}$, while $1.014 \%$ has been measured in ${ }^{57} \mathrm{Co}$ at $122 \mathrm{keV}$, showing that the HPGe detector works as expected.

\subsection{Nuclear resonant scattering system (NRSS)}

The nuclear resonant scattering system (NRSS) ${ }^{9}$ will be used to perform an absolute energy calibration of the CSPEC and GCAL subsystems. The underlying idea is to use a $\gamma$ counter to check (during an energy scan) whenever the beam overlaps one resonance level of a properly chosen target material, such as aluminium and boron for the LE line, and carbon for the HE one. The position of these levels (namely $E_{r}$ ) is well documented in the literature and their widths (on the order of fractions of an $\mathrm{eV}$ ) is smaller than the beam bandwidth. During the beam energy scan, an emission of photons of the same energy of the beam will be generated over the whole solid angle when the resonant 
condition is reached. In this case, the counting rate at the NRSS can be evaluated from the integrated cross-section as follows:

$$
\sigma_{\text {int }}=\int \sigma(E) d E=\sigma_{\max }^{0} \Gamma \pi / 2
$$

where $\sigma_{\max }^{0}$ is the resonant cross section at resonance energy $E=E_{r}$, and $\Gamma$ is the resonance width.

The NRSS ${ }^{10}$ of ELI-NP-GBC (Fig. 6) is an array of $5 \mathrm{~cm} x 5 \mathrm{~cm} \times 8 \mathrm{~cm}$ barium fluoride $\left(\mathrm{BaF}_{2}\right)$ crystals placed at an angle $\vartheta=135^{\circ}$ with respect to the beam axis to remove any background due to Compton scattering from the signal energy region. $\mathrm{BaF}_{2}$ was chosen because it is characterized by a fast scintillating component $(\tau \sim 0.6-0.8 \mathrm{~ns}$ peaked at $220 \mathrm{~nm}$ ), which is suitable for fast counting (FC mode) and timing purposes. The crystal array is equipped with a dual-readout system. The first readout is performed by a phototube with a Cs-Te photo-cathode coupled with a quartz window that enables selection of wavelengths from $160 \mathrm{~nm}$ to $320 \mathrm{~nm}$, thus eliminating most of the slow component of the signal. The second readout is devoted to the collection of the Cherenkov light produced inside the crystals. In the center of the array, a LYSO crystal (slower but with higher resolution with respect to $\mathrm{BaF}_{2}$ counters) allows a redundant measurement of the beam energy. When the system works as an energy spectrometer (ES mode), the $\mathrm{BaF}_{2}$ detectors surrounding the LYSO crystal are used as anti-Compton shields.

Many different resonance levels suitable for GBS operation have been individuated (results with a $0.25 \mathrm{~mm} \mathrm{Al}$ target are here shown) and their signals have been simulated in Geant4 together with possible background sources from inside and outside the beam line (Fig. 7). Photons with signal-like energy reach the NRSS $\sim 2$ ns earlier than that from resonant scattering, as studied in simulation. These sources of background can be eliminated thanks to the excellent timing performance of the counter. The combined readout of Cherenkov and scintillation light will be used to reduce any fake signal (e.g., arising from the residual slow scintillation) and the effects due to possible pile-up of lowenergy background photons.

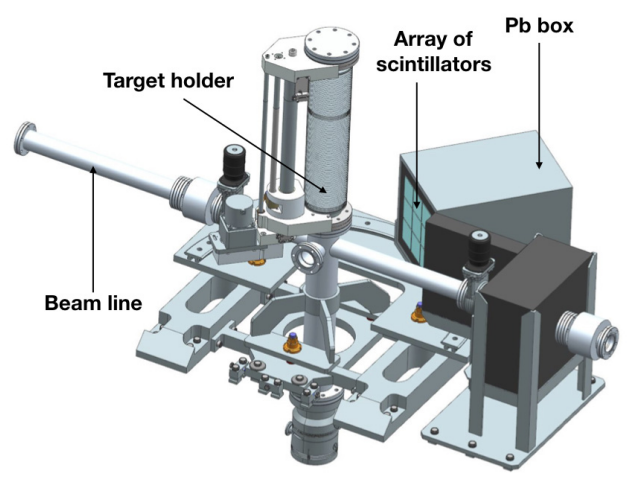

Fig. 6. Design of the NRSS. 


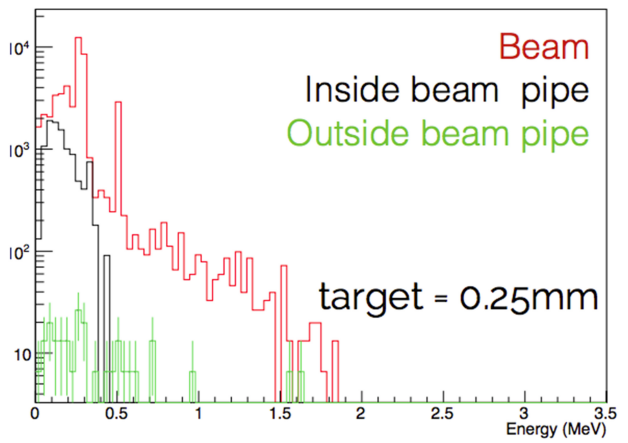

Fig. 7. Background on the NRSS for a $3 \mathrm{MeV}$ beam on a $0.25 \mathrm{~mm}$ Al target. Different sources of background are shown in the figure. In red: Compton backscattered photons from the target; in black: photons from outside the target but from inside the beam pipe (mainly scattered from the pipe walls nearby the target); in green: background from the surrounding material.

\subsection{Gamma beam profile imager (GPI)}

The gamma beam profile imager (GPI) measures the spatial distribution and uniformity of the beam and checks the correct alignment of the collimation system. To activate the GPI, a $0.5 \mathrm{~mm}$ LYSO screen will be moved on the beam axis by an actuator (the screen will be placed at a $45^{\circ}$ angle with respect to the beam). The light produced will be focused through a lens system onto a CCD camera that will be able to produce an image with a space resolution of $\sim 100 \mu \mathrm{m}$. The system will be hosted beyond the NRSS at $15.2 \mathrm{~m}$ from the interaction point for the HE line and $16.3 \mathrm{~m}$ for the LE line. At these distances, the transverse source image size is expected to be between $2.5 \mathrm{~mm}$ and $23 \mathrm{~mm}$, with uncertainty smaller than $2 \%$.

A Geant4 simulation for choosing the most promising scintillating material in terms of resolution, efficiency, and light yield was performed together with a Matlab raytracing code for the evaluation of the signal acquired by the CCD through lens system. The accuracy of these simulation tools has been cross-checked using radioactive sources (Fig. 8), and they have been used to simulate the response of the system to a realistic ELI-NP beam. For a 3-MeV beam, an exposure of a few seconds would be enough to give a well-resolved beam profile image. ${ }^{11}$

\subsection{Gamma sampling calorimeter (GCAL)}

The gamma sampling calorimeter (GCAL) was designed to evaluate the beam energy and intensity relying on the full $\gamma$-statistics. ${ }^{12}$ It uses a complementary approach to the energy determination provided by CSPEC, based on a destructive measurement. Therefore, it will be installed on a mobile platform and used only outside beam operation phases. 


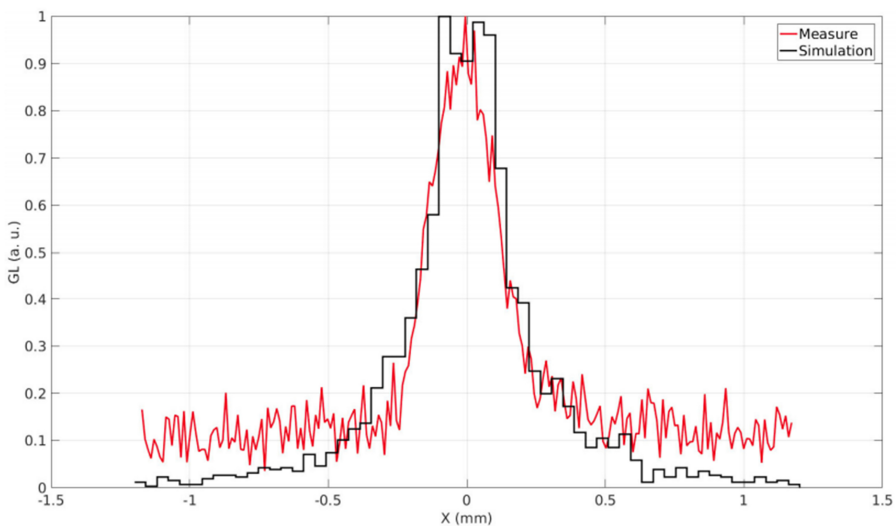

Fig. 8. Simulation and real measurement of the image produced by a 300 -second exposure of a ${ }^{241}$ Am source.
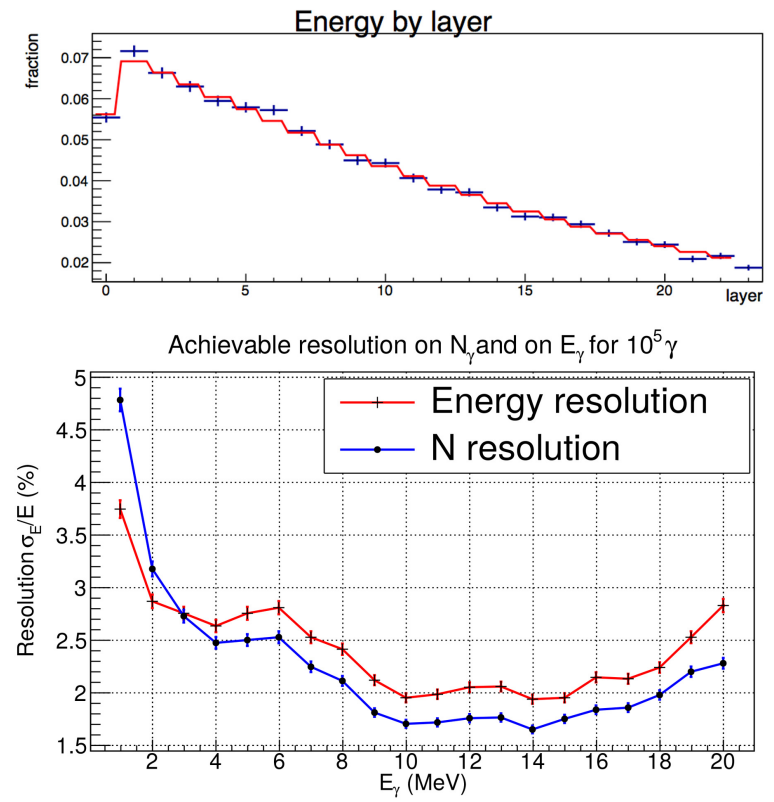

Fig. 9. (top) Measurement (blue) and fit (red) of deposited energy per layer to extract the average beam energy. (bottom) Simulation of energy and intensity resolution (in terms of number $\mathrm{N}$ of gammas impinging on GCAL).

The GCAL (based on sampling technique) is a 22-layer stack of 3-cm-thick polyethylene (PE) absorbers followed by thin Si strip-detector (300 $\mu \mathrm{m}$ thick) layers. Each layer consists of seven Si-strip modules of the same radiation-hard technology developed by Hamamatsu for the CMS experiment. ${ }^{13}$ Its main characteristics are as 
follows:

- Low-Z absorber; the total interaction cross-section for these materials decreases monotonically as a function of the $\gamma$-energy. Due to this behavior, the average depth of photon interaction will increase with increasing energy, providing a longitudinal shower profile that can be uniquely related to the beam energy;

- Fast signal collection to fit with the time structure of the ELI-NP $\gamma$-beam.

The PE has been chosen among other low $\mathrm{Z}$ materials since it is inexpensive and easily workable. The choice of longitudinal and transverse sizes was driven by Monte Carlo simulations of signal fluctuation. A per-layer profile parameterization was obtained from simulations at different energies having the spectrum peak energy as the only parameter. A fit of data on these templates can then be used to unambiguously infer the average beam energy (Fig. 9, top). Combining this measurement with the total energy deposit, given the quasi-monochromaticity, the beam intensity can be extracted. Figure 9 (bottom) shows the energy and intensity resolution obtained in simulations of a single micro-pulse ( $10^{5}$ photons) at different energies. A low-percentage resolution can be achieved throughout the energy range. These uncertainties drop below $0.1 \%$ after collecting $\sim 10^{3}$ pulses corresponding to a 3 -second data taking. Tests conducted on prototype boards have validated the capacity to separate signals from different micropulses.

\section{Conclusions}

The collimation system of ELI-NP-GBS will play a fundamental role in the definition of the final bandwidth and intensity of the $\gamma$-beam. Given the unprecedented working condition of the beam, the design and setup of the beam-characterization system is a complex experimental challenge. All the subsystems have been designed and optimized using detailed simulations and are now in final phase of testing in the three WP09 sites Catania, Ferrara and Florence.

\section{References}

1. Eli-NP users community, The Eli-NP White Book, www.eli-np.ro/documents/ELI-NPWhiteBook.pdf.

2. C.A. Ur et al., Nucl. Instr. Meth. Phys. Res. B355, 198 (2015).

3. D. Filipescu et al., Eur. Phys. J. A51, 185 (2015).

4. O. Adriani et al., Technical Design Report EuroGammaS proposal for the ELI-NP Gamma Beam System, arXiv:1407.3669.

5. P. Cardarelli et al., Nucl. Instr. Meth. Phys. Res. B355, 237 (2015).

6. K. Yokoya. User Manual of CAIN, version 2.40., http://lcdev.kek.jp/yokoya/CAIN/ Cain242/CainMan242.pdf (2009).

7. G. Paternò et al., Nucl. Instr. Meth. Phys. Res. B402, 349 (2017). 
8. M. Gambaccini et al., Gamma Beam Characterization Design Report, EuroGammaS deliverables report - D081 (2015).

9. F.R. Metzger, Progr. Nucl. Phys. 7, 53 (1959).

10. M.G. Pellegriti et al., Nucl. Instr. Meth. Phys. Res. A865, 60 (2016).

11. P.Cardarelli et al., Nucl. Instr. Meth. Phys. Res. A893, 109 (2018).

12. M. Lenzi et al., J. Inst. 12.02, C02051 (2017).

13. L. Borrello et al., Sensor Design for the CMS Silicon Strip Tracker, CMS note 2003/020. 